\title{
The Combination Therapy of Fluorouracil and Oxaliplatin Suppress the Progression of Colon Cancer Through miR-I83-5p/SOCS3 Axis and Downregulating PD-LI
}

This article was published in the following Dove Press journal: Cancer Management and Research

\author{
Changling Tu' \\ Yufeng Wang' \\ Xianshuo Cheng (D) $^{2}$ \\ Ying Zhu' \\ Wenli Yuan ${ }^{3}$ \\ Jian Dong ${ }^{4}$
}

'Department of Cadres Medical Oncology, The Third Affiliated Hospital of Kunming Medical University, Yunnan Cancer Hospital, Yunnan Cancer Center, Kunming, 650I 18, People's Republic of China; ${ }^{2}$ Department of Colorectal Surgery, Third Affiliated Hospital, Kunming Medical University, Kunming, 650II8, Yunnan, People's Republic of China; ${ }^{3}$ Department of Clinical Laboratory, The Fourth Affiliated Hospital of Kunming Medical University, Kunming, 65002I, Yunnan, People's Republic of China; ${ }^{4}$ The Third Affiliated Hospital of Kunming Medical University, Yunnan Cancer Hospital, Yunnan Cancer Center, Kunming, 650II8, People's Republic of China
Correspondence: Jian Dong

The Third Affiliated Hospital of Kunming Medical University, Yunnan Cancer Hospital, Yunnan Cancer Center, No. 519 Kun Zhou Road, Xishan District, Kunming, 650II8, Yunnan, People's

Republic of China

Tel +86-87I-68I79549

Email3196259175@qq.com
Purpose: The purpose of this study was to investigate the mechanism of combination of fluorouracil (FU) and oxaliplatin (OXA) on the progression of colon cancer via miR-183-5p/ SOCS3 axis and regulating PD-L1.

Methods: HCT116 cells were treated with $4 \mu \mathrm{M}$ OXA and $10.5 \mu \mathrm{M}$ FU, or exogenous regulation of the expression of miR-183-5p, SOCS3 and PD-L1 in HCT116 cells. CCK-8 assay was employed to detect cell viability of HCT116 cells. Flow cytometry was performed to assess the apoptosis and cell cycle. The expression level of SOCS3, PD-L1, chemokines (CCL1, CCL4 and CCL7) and immune escapes related proteins (EGFR, STARD1 and STARD3) in HCT116 cells were assessed by Western blotting. In addition, dual-luciferase reporter gene was carried out to verify the targeted relationship between miR-183-5p with SOCS3.

Results: Our study demonstrated that the combination of OXA and FU remarkably suppressed proliferation, promoted apoptosis and arrest cells in G0/G1 phrase of HCT116 cells, and observably downregulated the expression of PD-L1, CCL1, CCL4, CCL7, EGFR, STARD1 and STARD3. Meanwhile, the combination of OXA and FU significantly downregulated miR-183-5p expression. Knockdown of miR-183-5p also repressed the proliferation, promoted apoptosis and arrest cells in G0/G1 phrase of HCT116 cells, and downregulated the expression of PD-L1, CCL1, CCL4, CCL7, EGFR, STARD1 and STARD3. In addition, our study proved that miR-183-5p upregulated PD-L1 by targeting downregulated SOCS3 expression. Finally, we demonstrated that the combination therapy of OXA and FU inhibited the proliferation, promote apoptosis and arrest cells in G0/G1 phrase by downregulating PD-L1 via miR-183-5p/SOCS3 axis.

Conclusion: The combination therapy of OXA and FU could suppress the malignant biological behavior, and the mechanism was realized by inhibiting PD-L1 through miR$183-5 \mathrm{p} / \mathrm{SOCS} 3$ axis.

Keywords: colon cancer, combination therapy, PD-L1, miR-183-5p, SOCS3

\section{Introduction}

Colorectal cancer (CRC) is one of the most common cancers in the digestive system with a high incidence. Improved perioperative management and adjuvant therapy increased survival in patients with early CRC. ${ }^{1}$ However, patients with advanced $\mathrm{CRC}$ still have a higher risk of recurrence after surgery. ${ }^{2}$ At present, with the development of immune-oncology, it is gradually recognized that targeted PD1/PD-L1 therapy 
may be a new direction in the treatment of CRC. ${ }^{3,4}$ We aimed to investigate the effect of combined treatment with fluorouracil (FU) and oxaliplatin (OXA) on expression of PD1.

PD-L1 is encoded by the CD274 gene which is an immune inhibitory molecule involved in immune regulation. ${ }^{5}$ PD-L1 represses the activation of $\mathrm{CD} 8+$ $\mathrm{T}$ cells in lymph nodes through binding to programmed cell death receptor 1 (PD1). ${ }^{6,7}$ The mechanism of PD1/PDL1 maintain immune homeostasis under physiological conditions, whereas cancer cells evade immune surveillance using this mechanism. Meanwhile, Llosa et al reported that suppression of the interaction between PDL1 and PD1 could restore anti-tumor immunity. ${ }^{8}$ However, the mechanism that regulates PD-L1 is still unclear.

FU and OXA are commonly used chemotherapy drugs following surgical resection for CRC. It has been reported that FU mainly effects on the TS and DPYD pathways. ${ }^{9}$ And OXA causes intrastrand and interstrand DNA-platinum adducts and then repress gene transcription by segregation of transcription factors or bring about arrest of $\mathrm{G} 2 / \mathrm{M}$ stage. ${ }^{10}$ In the preliminary experiment, we screened key proteins and miRNAs with significant effects on the PD1/ PD-L1 pathway via CHIP experiments. We found that miR183-5p and SOCS3 were most significant before and after disposal of FU and OXA, and SOCS3 is a key target gene of miR-183-5p. Many studies had confirmed that miR-183-5p was closely related to process of CRC. For instance, the cellular activity and invasiveness of CRC cells were reduced by mir-183-5p knockdown. ${ }^{11}$ miR-183-5p enhances the radio resistance of $\mathrm{CRC}$ via targeting ATG5. ${ }^{12}$ SOCS3 plays an anti-cancer role in breast cancer, ${ }^{13}$ lung cancer, ${ }^{14}$ CRC, ${ }^{15}$ and so on. For example, SOCS3 was downregulated in triple-negative breast cancer, overexpression of SOCS3 result in a less aggressive oncogenic phenotype. ${ }^{13}$ Also, Wang et al have shown that SOCS3 upregulation promoted cell apoptosis and suppressed proliferation of pancreatic cancer SW1990 cells. ${ }^{16}$ In our article, our goal was to investigate the impact of the combination of FU and OXA on the expression of PD-L1 and malignant biological behavior of CRC HCT116 cells via miR-183-5p/SOCS3 axis.

\section{Materials and Methods}

\section{Tissue Samples}

Tissue specimens were obtained from CRC patients who were admitted to Yunnan Cancer hospital from August 2018 to December 2019 (the distance from the tumor margin is greater than $5 \mathrm{~cm}$ ). A total of 40 patients with CRC had an average age of $56.25 \pm 12.64$ years. All patients were confirmed by histopathological examination. The patients did not receive chemotherapy or radiotherapy before surgery, and the case data were complete. All tissue samples were collected and temporarily stored in liquid nitrogen, and transferred to $-80^{\circ} \mathrm{C}$ refrigerator after 24 hours. This research is based on the Declaration of Helsinki. This study has been approved by the ethics committee of Yunnan Cancer hospital, and all participants have signed the informed consent.

\section{Cell Culture, Transfection and Treatment} HCT116 cells is maintained with DMEM medium (Gibco, USA), which contain 10\% FBS (Gibco, USA), $0.1 \mathrm{U} / \mathrm{mL}$ penicillin and $0.1 \mu \mathrm{g} / \mathrm{mL}$ streptomycin, and maintained at $37^{\circ} \mathrm{C}$ in a $5 \% \mathrm{CO} 2$ incubator (Thermo Fish Scientific, USA). Cells treated with $4 \mu \mathrm{M}$ OXA or $10.5 \mu \mathrm{M}$ FU.

miR-183-5p mimics, inhibitors or scramble (gemma, Shanghai, China), miR-183-5p inhibitor (gemma, Shanghai, China), si-SOCS3 (Promega, USA), pcDNA3.1-SOCS3 (Promega, USA) and si-PD-L1 (Promega, USA) were transfected into HCT116 cells according to manufacturer's instructions.

\section{RT-qPCR Analysis}

OXA and FU treated and stably transfected cells were collected and total RNA was leached from each group employing Trizol reagent (Promega, USA). The TaqMan microRNA reverse transcription kit (Millipore, USA) is used to produce cDNA. The relative expression was analyzed using $2^{-\Delta \Delta \mathrm{Ct}}$ method by normalizing to U6 expression.

\section{CCK-8 Assays}

OXA and FU treated and stably transfected cells were collected and digested with trypsin. $4 \times 10^{3}$ cells were inoculated into 96-well plates (Corning, USA) and added $200 \mu \mathrm{L}$ medium for each well. Cell proliferation activity was measured according to the instructions of the CCK-8 kit. The absorbance was measured using the enzyme marker at $450 \mathrm{~nm}$.

\section{Flow Cytometry}

Cells apoptosis and cycle were detected by flow cytometry (Thermo Fish Scientific, USA). HCT116 cells were digested with trypsin and resuspended in the $1 \times$ binging buffer. $5 \mu \mathrm{L}$ Annexin v-fitc and PI reagents were added with a proportion of $5 / 1$ and placed in darkness for $20 \mathrm{~min}$. 
HCT116 cells were digested with trypsin and incubated overnight with $70 \%$ ice ethanol at $4^{\circ} \mathrm{C}$. The next day, PBS containing RNA enzymes was added, and incubated at $37^{\circ}$ C for $30 \mathrm{~min}$. After incubation with PI for $15 \mathrm{~min}$ in dark, the cells were dyed at room temperature for $30 \mathrm{~min}$. Finally, the cell cycle was identified using flow cytometry.

\section{Western Blotting Analysis}

Total proteins from tissues and cells were extracted for Western blot analysis. The total protein in each group of tissues and cells was leached with RIPA lysate (Bio-Rad, USA). BCA method was employed to assess the protein concentration. SDS-PAGA was employed to separate the protein bands, and then transferred onto PVDF membranes (Thermo Fish Scientific, USA). The PVDF membranes were incubated in $5 \%$ defatted milk at $37^{\circ} \mathrm{C}$ for 2 h. Next, the primary antibody (Abcam, UK) of the target protein was added and incubated at $4^{\circ} \mathrm{C}$ overnight. PBST was rinsed three times and HRP-labeled secondary antibody (Abcam, UK) was added. Subsequently, the membranes were identified with the ECL plus Western blotting detection reagents. Finally, the gel imager was used for observation and photography, and Image $\mathrm{J}$ was performed for quantitative analysis of the bands.

\section{Immunohistochemical}

After dewaxing, hydration and antigen repair, tissue paraffin sections were soaked in 3\% hydrogen peroxide solution for $15 \mathrm{~min}$ and rinsed with PBS 3 times. Then bovine serum albumin (BSA) was sealed for $15 \mathrm{~min}$ and incubated overnight with the primary antibody at $4{ }^{\circ} \mathrm{C}$.
The next day, a secondary antibody was incubated for $30 \mathrm{~min}, \mathrm{DAB}$ was used for color development, and hematoxylin was washed with tap water for $5 \mathrm{~min}$ after staining, the slices were dehydrated and sealed, and finally observed and photographed under a microscope.

\section{Luciferase Assay}

Starbase was used to evaluate the target sequences of mIR$183-5 p$ and SOCS3. SOCS3 wild sequence or mutant sequence within miR-183-5p binding sites was insert into pmirGLO plasmids. The HEK-293T cells of pmirGLO/ SOCS3-WT and pmirGLO/SOcs3-MUT were transfected with miR- mimics or miR-NC, respectively. The dualluciferase reporter gene kit (Solarbio, Beijing, China) was used to assess the luciferase activity.

\section{Statistical Analysis}

SPSS 22.0 is performed for statistical analysis and the dates were shown as $\mathrm{x} \pm \mathrm{s}$. The LSD test was used for comparison between the two groups. A one-way ANOVA was used for comparison of multiple groups. $P<0.05$ showed a statistically significant difference.

\section{Results}

\section{Decreased Expression of SOCS3 is Associated with the Progression of Colorectal Cancer}

The results of immunohistochemical (IHC) staining showed that the expression of SOCS3 was weakly positive
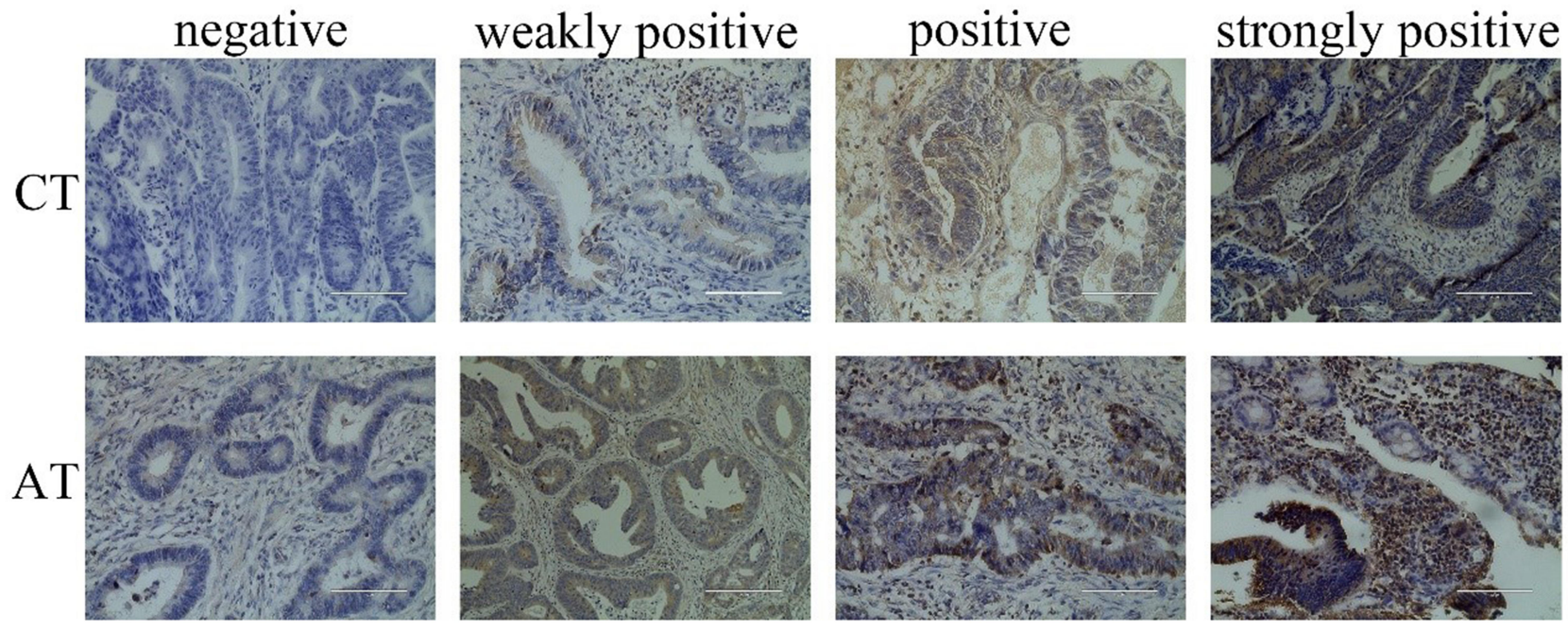

Figure I Immunohistochemical was used to detect of SOCS3 expression in cancer tissues and adjacent tissues of CRC patients. 
in 24 cases $(60 \%)$ and negative in only 1 case $(2.5 \%)$ of the 40 cancer tissue samples. However, only 7 (17.5\%) of the control tissue samples were weakly positive. SOCS3 is mainly located in the cytoplasm, with occasional partial cell membrane staining, and the positive staining is tawny $(\mathrm{P}<0.05$, Figure 1).

According to the IHC staining result of SOSC3 in CRC cancer tissue, 40 patients were divided into a high group (16 cases) and a low group (24 cases). As shown in Table 1, the expression of SOCS3 was not correlated with age, gender, differentiation, tumor size, of patients, and the difference was not statistically significant $(\mathrm{P}>$ 0.05). However, it was significantly correlated with the TNM stage, lymph nodes and distant metastasis of patients $(\mathrm{P}<0.05)$.

Table I Correlation Between SOCS3 Expression and Pathological Characteristics of CRC Cancer Patients

\begin{tabular}{|c|c|c|c|c|c|}
\hline \multirow[t]{2}{*}{$\begin{array}{l}\text { Clinicopathological } \\
\text { Features }\end{array}$} & \multirow[t]{2}{*}{$\mathbf{N}$} & \multicolumn{2}{|c|}{$\begin{array}{l}\text { Expression } \\
\text { Level of WSB2 }\end{array}$} & \multirow[t]{2}{*}{$X^{2}$} & \multirow[t]{2}{*}{ P-value } \\
\hline & & $\begin{array}{l}\text { High } \\
\text { Level } \\
(n=16)\end{array}$ & $\begin{array}{l}\text { Low } \\
\text { Level } \\
(n=24)\end{array}$ & & \\
\hline $\begin{array}{l}\text { Ages (years) } \\
\quad<50 \\
\quad \geq 50\end{array}$ & $\begin{array}{l}19 \\
21\end{array}$ & $\begin{array}{l}7 \\
9\end{array}$ & $\begin{array}{l}12 \\
12\end{array}$ & 0.198 & 0.824 \\
\hline $\begin{array}{l}\text { Gender } \\
\text { Male } \\
\text { Female }\end{array}$ & $\begin{array}{l}21 \\
19\end{array}$ & $\begin{array}{l}8 \\
8\end{array}$ & $\begin{array}{l}13 \\
11\end{array}$ & 0.219 & 0.805 \\
\hline $\begin{array}{l}\text { Differentiation status } \\
\text { Highly or } \\
\text { moderately } \\
\text { differentiated } \\
\text { Poorly } \\
\text { differentiated }\end{array}$ & 17 & 7 & $\begin{array}{l}14 \\
10\end{array}$ & 0.158 & 0.894 \\
\hline $\begin{array}{l}\text { Tumor diameter } \\
\begin{array}{l}\geq 3 \mathrm{~cm} \\
<3 \mathrm{~cm}\end{array}\end{array}$ & $\begin{array}{l}19 \\
21\end{array}$ & $\begin{array}{l}8 \\
8\end{array}$ & $\begin{array}{l}11 \\
13\end{array}$ & 0.692 & 0.657 \\
\hline $\begin{array}{l}\text { TNM stage } \\
\text { I-II } \\
\text { III-IV }\end{array}$ & $\begin{array}{l}16 \\
24\end{array}$ & $\begin{array}{l}10 \\
6\end{array}$ & $\begin{array}{l}6 \\
17\end{array}$ & 4.793 & $0.039 *$ \\
\hline $\begin{array}{l}\text { lymph node } \\
\text { metastasis } \\
\text { Yes } \\
\text { No }\end{array}$ & $\begin{array}{l}10 \\
30\end{array}$ & $\begin{array}{l}2 \\
12\end{array}$ & $\begin{array}{l}8 \\
16\end{array}$ & 5.492 & $0.027^{*}$ \\
\hline
\end{tabular}

Note: $* \mathrm{P}<0.05$.

\section{The Combination of FU and OXA}

\section{Suppressed the Malignant Biological}

Behavior of HCTII 6 Cells

In order to verify the impact of combination therapy of FU and OXA on the malignant biological behavior of CRC cells, HCT116 cells were treated with $4 \mu \mathrm{M}$ OXA or $4 \mu \mathrm{M}$ OXA combined with $10.5 \mu \mathrm{M}$ FU. Cell viability of HCT116 cells at 24, 48, 72 and $96 \mathrm{~h}$ were detected by CCK-8 assay, and cell viability of OXA group was suppressed $(P<0.01)$, while in OXA and FU combined treatment group was lower $(P<0.001$, Figure 2A). Flow cytometry was undertaken on cell apoptosis and cell cycle. The remarkable rise of apoptosis of HCT116 cells in OXA group compared to the control group $(P<0.01)$, while the apoptosis percentage in OXA and FU combined treatment group significantly greater $(P<0.01$, Figure $2 \mathrm{~B})$. Compared with the control group, OXA or OXA combined with FU dramatically arrested cells in G0/G1 phase (all $P<0.01$, Figure 2C). On the other hand, we also examined the impact of OXA and FU on the expression levels of chemokines (CCL4, CCL4 and CCL7), immune-related proteins (EGFR, STARD1 and STARD3) and PD-L1 by Western blotting. As shown in Figure 2D of OXA dramatically repressed the expression of CCL4, CCL4, CCL7, EGFR, STARD1, STARD3, as well as PDL1 $(P<0.05, P<0.01$ or $P<0.001)$. At the same time, OXA and FU combined treatment group had a more significant effect. OXA and FU inhibited the malignant biological behavior of HCT116 cells and the mechanism maybe achieved by regulating PD-L1 expression.

\section{FU Combined with OXA Significantly Downregulated miR-183-5p in Colon Cancer Cells}

The differences expression miRNA in the negative control group, OXA treated group, OXA and FU combined treatment were screened by miRNA microarray. miRNAs who was significantly reduced in OXA treatment group, OXA and FU combined treatment group were selected for subsequent analysis. miR-183-5p was significantly downregulated with single or double treatment (Figure 3A). Next, we exposed HCT116 cells to $4 \mu \mathrm{M}$ OXA or $4 \mu \mathrm{M}$ OXA combined with $10.5 \mu \mathrm{M}$ FU for $48 \mathrm{~h}$. RT-qPCR was performed to test miR-183-5p expression. The results suggested that miR-183-5p expression was notably downregulated in the OXA group and OXA and FU 


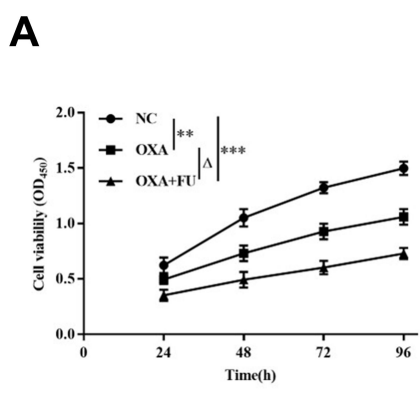

C

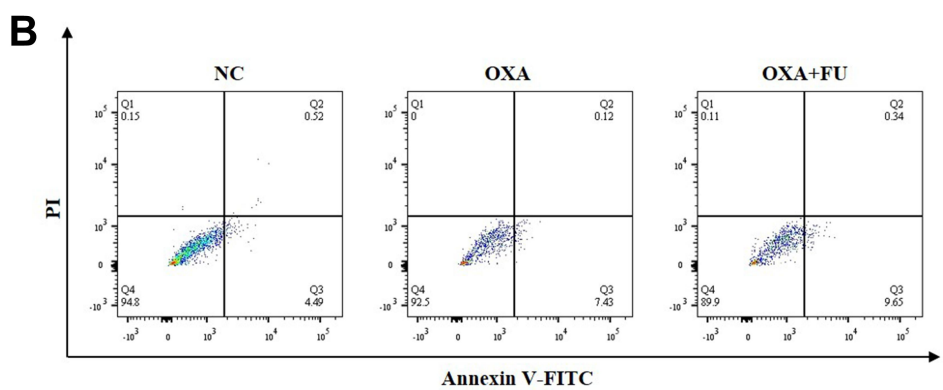

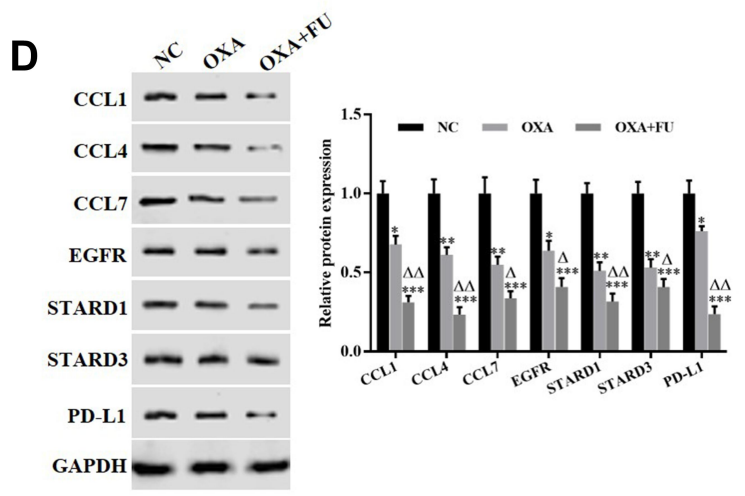

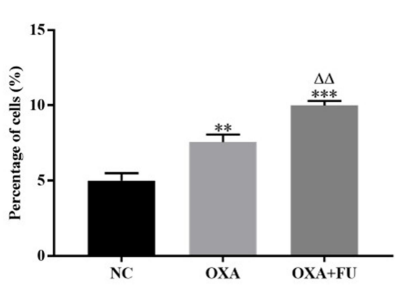

Figure 2 The combination of FU and OXA suppressed the malignant biological behavior of HCTII6 cells and the expression of PD-LI. (A) CCK-8 assay was performed to detect the proliferation of HCTII6 cells. (B and C) Flow cytometry was employed to assess the apoptosis and cell cycle of HCTII6 cells. (D) Western blotting was carried

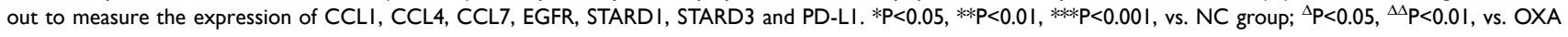
treated group.

combined treatment group compared with the control group (Figure 3B). The results of RT-qPCR were consistent with chip result. However, the change degree of relative expression level was obviously weaker than that of chip. According to the differential expression results of miRNA, we believe that the down-regulation of miR-183$5 \mathrm{p}$ may be one of the key factors for the combined treatment of OXA and FU to inhibit the malignant progression of CRC cells. Therefore, miR-183-5p was selected for subsequent experiments. Next, the target genes of miR183-5p were analyzed and the signaling pathways of the target genes were enriched, and the results showed that the target genes of miR-183-5p participated in and regulated the JAK-stat pathway (Figure 3C). Therefore, SOCS3, an inhibitor of the JAK-stat pathway, was selected as the target gene of miR-183-5p (Figure 3D).

\section{Silencing miR-183-5p Repressed the Malignant Biological Behavior of HCTII6 Cells}

The transfection efficiency was shown in Figure 4A, miR$183-5 p$ is observably downregulated in miR-183-5p inhibitor group $(P<0.001)$. CCK-8 assay demonstrated that knockdown of miR-183-5p notably suppressed cell viability of
HCT116 cells $(P<0.01$, Figure 4B). Flow cytometry consequences indicted that the apoptosis percentage of HCT116 cells increased after treatment with miR-183-5p inhibitor compared to the control group $(P<0.01$, Figure 4C). Cells is arrested in G0/G1 phase by knockdown of miR-183-5p $(P<0.01$, Figure 4D). Moreover, Western blotting consequences indicated that the expression of CCL4, CCL4, CCL7, EGFR, STARD1, STARD3 and PD-L1 in miR-183$5 \mathrm{p}$ inhibitor group were dramatically downregulated relative to the level in the control group (Figure 4E). Thus, these data manifested knockdown of miR-183-5p suppressed the malignant biological behavior of HCT116 cells.

\section{miR-183-5p Targeted Downregulates the Expression of SOCS3}

The Starbase database indicates that SOCS3 targets miR183-5p (Figure 5A). We discovered that co-transfection of miR-183-5p mimics notably decreased the WT SOCS3 luciferase intensity $(P<0.01)$, whereas the luciferase intensity was not reduced when the cells were transfected with a mutated SOCS3 reporter (Figure 5B). On the other hand, Western blotting results indicated that the expression level of SOCS3 was downregulated in miR-183-5p mimics group relative to the level in the control group $(P<0.01$, 
A
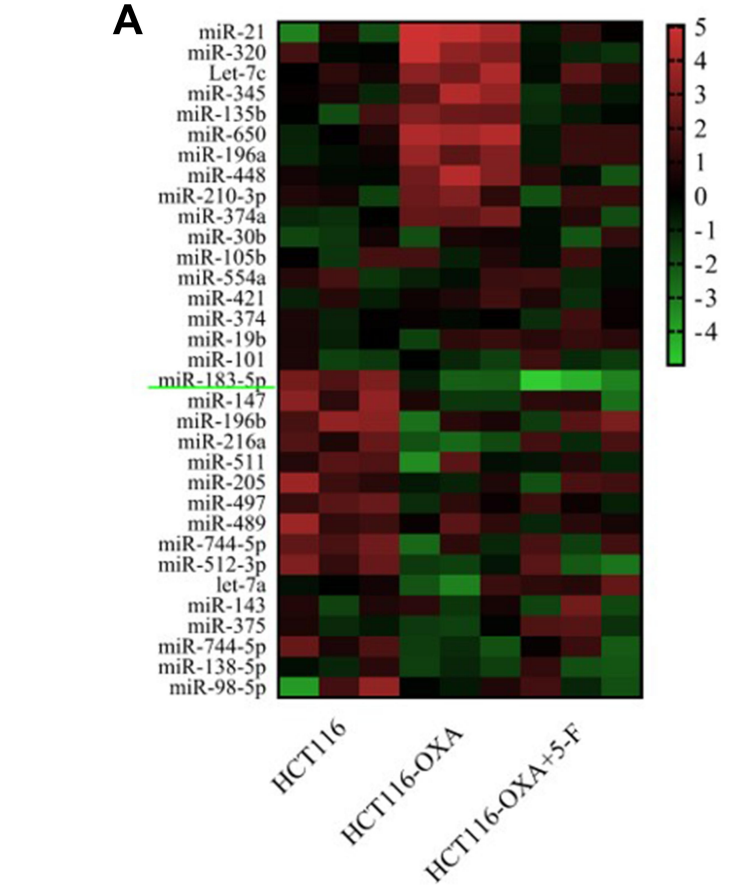

C

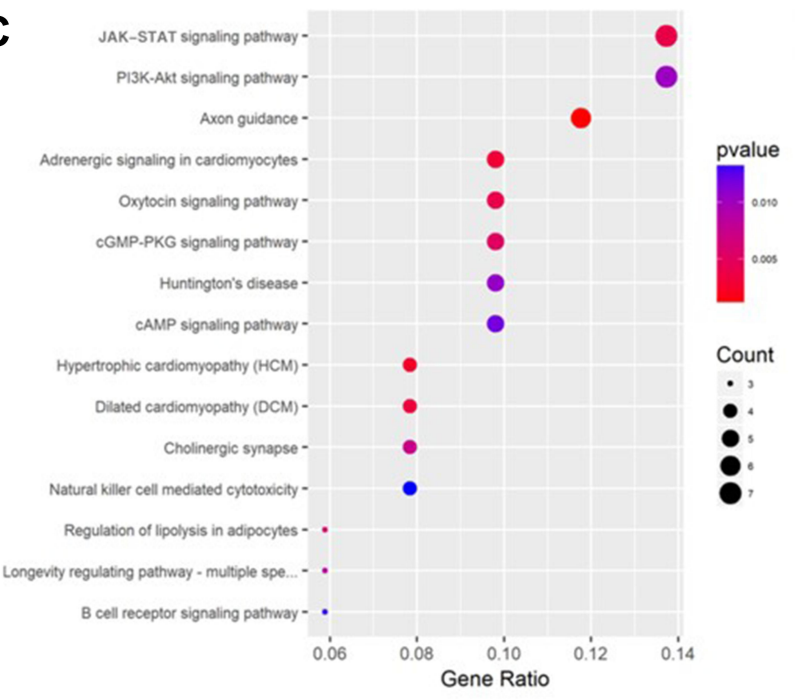

B

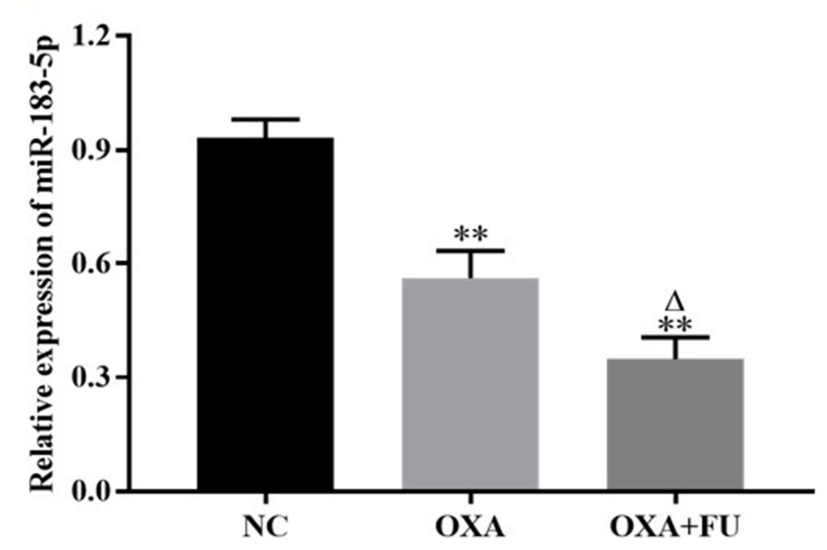

D

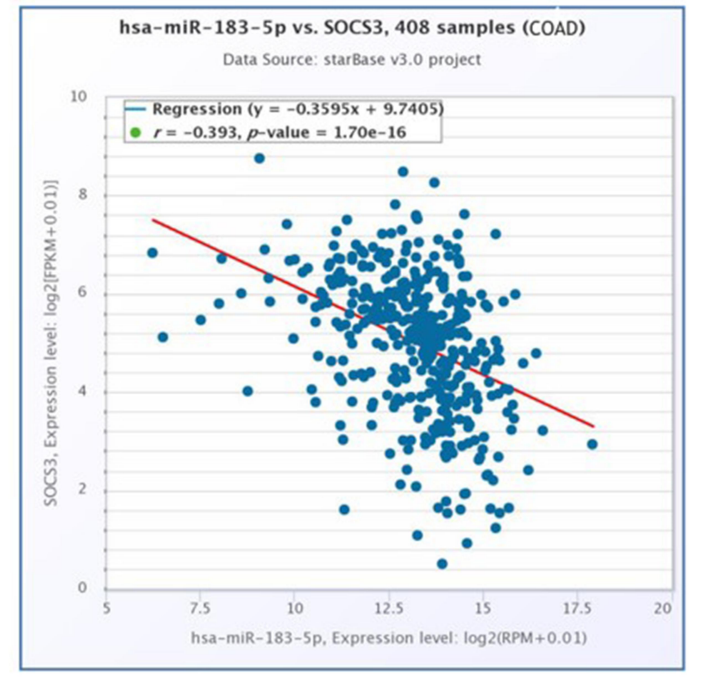

Figure $3 \mathrm{Fu}$ and OXA downregulated the expression of miR-183-5p. (A) Heat map is used to show the differential expression of miR-183-5p in CRC cells of control group, OXA group, and OXA+5-F group. (B) qRT-PCR was used to verify the down-regulated expression of miR-183-5p in colon cancer cells with OXA and FU+OXA group. (C) Pathway enrichment results of miR-I83-5p target genes. (D) miR-I83-5p was negatively correlated with SOCS3 expression in colon cancer. $* * P<0.0 \mathrm{I}$, vs. NC group; ${ }^{\Delta} \mathrm{P}<0.05$, vs. OXA treated group.

Figure 5C). These consequences demonstrated that miR183-5p targeted the downregulated SOCS3 expression.

\section{miR-I83-5p Upregulated PD-LI Expression by Downregulating SOCS3}

To demonstrate whether the expression level of PD-L1 was regulated by miR-183-5p/SOCS3 axis, pcDNA3.1-SOCS3 and miR-183-5p mimics were transfected into HCT116 cells. The results pointed out that overexpression of SOCS3 observably upregulated the expression of SOCS3 $(P<0.05)$ and reduced the expression of PD-L1 compared to the control group $(P<0.001)$, whereas overexpression of miR-183-5p and SOCS3 simultaneously restored the expression SOCS3 and PD-L1 (Figure 6A and B). 


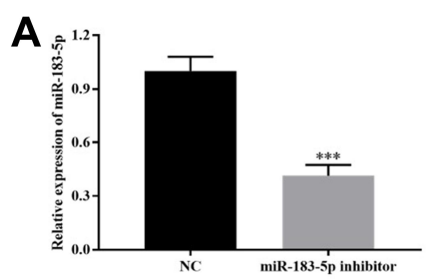

\section{B}

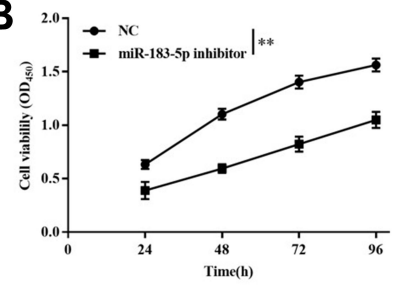

C

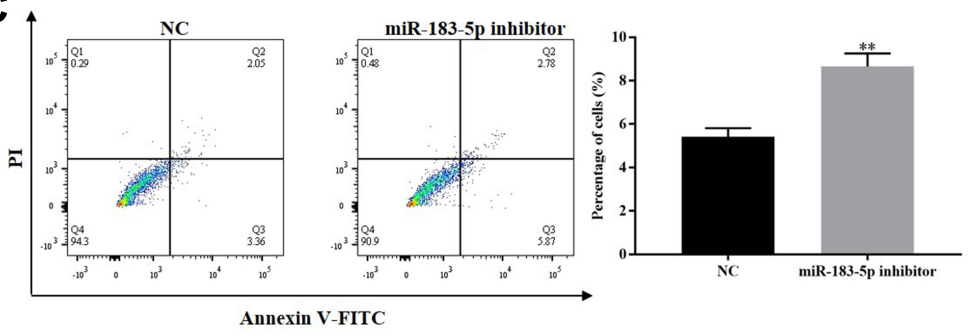

D

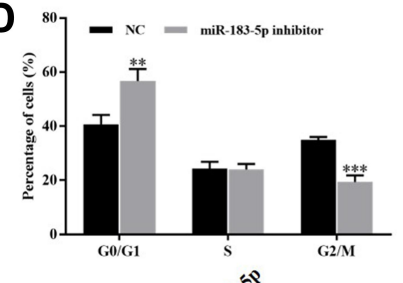

E

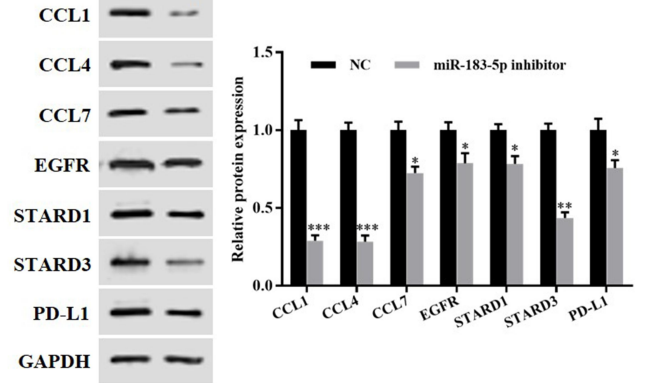

Figure 4 Silencing miR-183-5p inhibited the malignant biological behavior of HCTII6 cells. (A) RT-qPCR was used to detect the expression of miR-I83-5p. (B) CCK-8 assay was performed to detect the proliferation of HCTII 6 cells. (C and D) Flow cytometry was employed to assess the apoptosis and cell cycle of HCTII6 cells. (E) Western blotting was carried out to measure the expression of CCLI, CCL4, CCL7, EGFR, STARDI, STARD3 and PD-LI. *P<0.05, **P<0.0I, ***P<0.00I, vs. NC group.

A

Target: 5' CCCGGGUGCUCCCGCUCUGCCAUa 3'

\section{|::| || ||: ||||||}

miRNA : 3' ucaCUUAAGAUGGU--CACGGUAu 5'

B

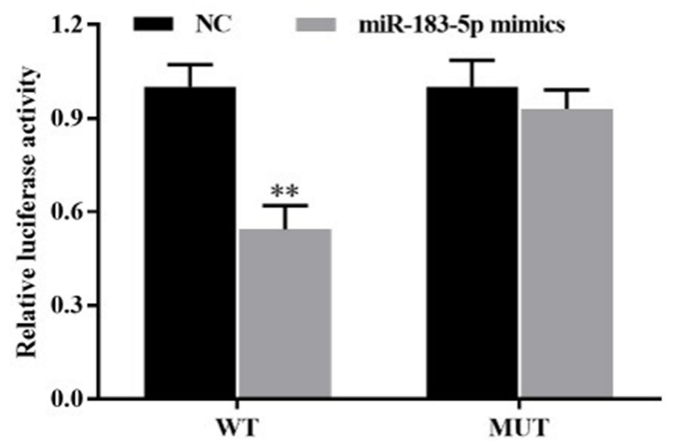

C
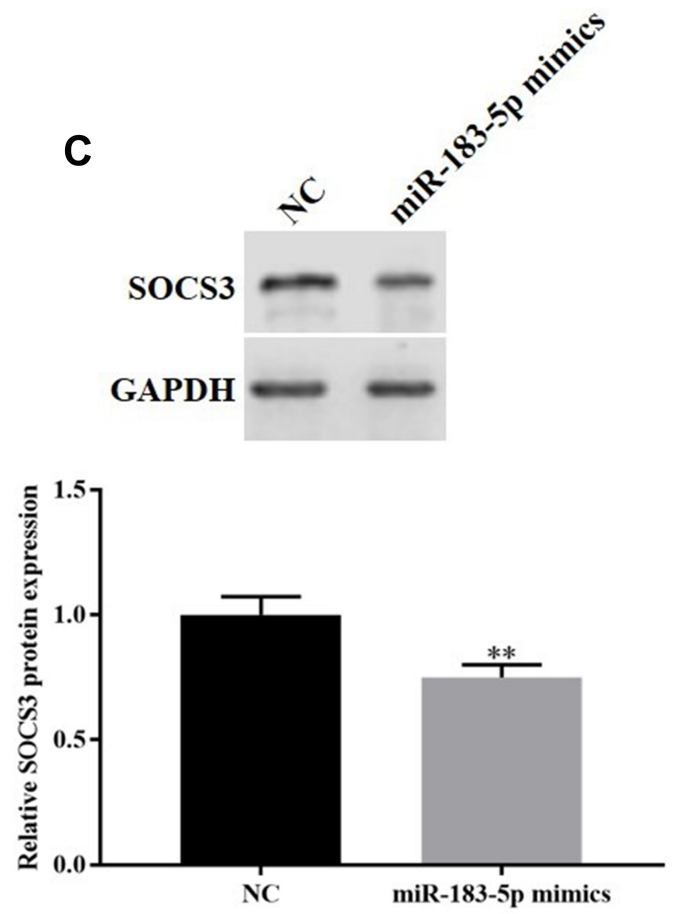

Figure 5 miR-183-5p targeted downregulate the expression of SOCS3. (A) Starbase database was used to predict the sequences between miR-I83-5p with SOCS3. (B) Dual-luciferase reporter gene was carried out to verify the targeted relationship between miR-I83-5p with SOCS3. (C) Western blotting was used to detect the expression of SOCS3. $* * \mathrm{P}<0.01$, vs. NC group. 

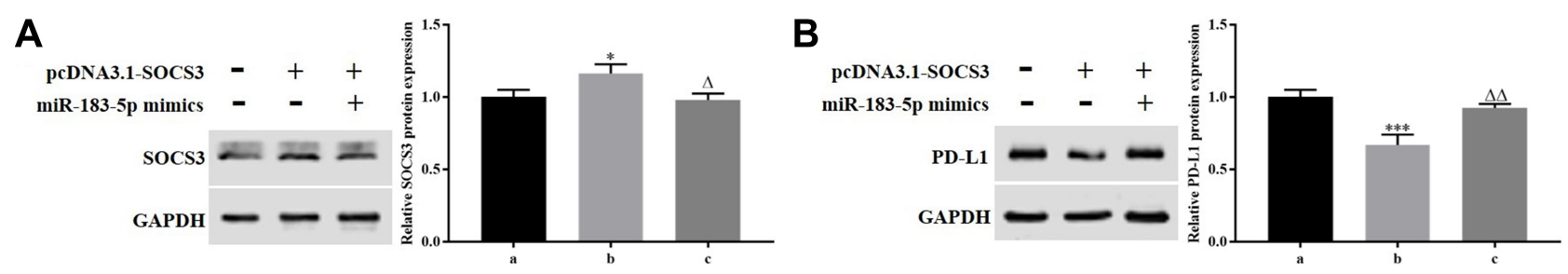

Figure 6 miR-183-5p upregulated PD-LI expression by downregulating SOCS3. (A and B) Western blotting was performed to measure the expression of SOCS3 and PD-

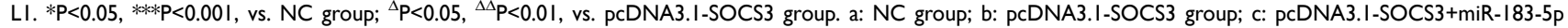
mimics group.

\section{Combination Therapy of FU and OXA Inhibited the Malignant Biological Behavior of HCTII6 Cells by Downregulating PD-LI via miR-183-5p/ SOCS3 Axis}

To determine the effect of combination of FU and OXA on the malignant biological behavior of HCT116 cells by downregulating PD-L1 via miR-183-5p/SOCS3 axis. The cells were divided into a control group, an si-SOCS3 group, an si-SOCS3 + miR-183-5p inhibitor group, an siSOCS3 + si-PD-L1 group and an si-SOCS3 + FU + OXA group. SOCS3 expression was signally downregulated in si-SOCS3 group compared to the control group $(P<0.001)$, while other groups restored the expression of SOCS3
(Figure 7A). CCK-8 assay proved that silencing SOCS3 dramatically promoted the proliferation of HCT116 cells $(P<0.01$ or $P<0.001$ Figure $7 \mathrm{~B})$. Flow cytometry results indicated that knockdown of SOCS3 significantly suppressed the apoptosis of HCT116 cells $(P<0.001)$, as well as promoted cells in $\mathrm{G} 2 / \mathrm{M}$ phase $(P<0.01$, Figure $7 \mathrm{C}$ and D). Finally, we performed Western blotting for detecting chemokines and immune escape related proteins. Knockdown of SOCS3 significantly upregulated the expression of CCL4, CCL4, CCL7, EGFR, STARD1, STARD3 and PD-L1 $(P<0.01$, Figure 7E). At the same time, si-SOCS3 + miR-183-5p inhibitor, si-SOCS3 + siPD-L1 or si-SOCS3 + FU + OXA restored the facilitation effect of silencing SOCS3 on the malignant biological behavior of HCT116 cells. These above results
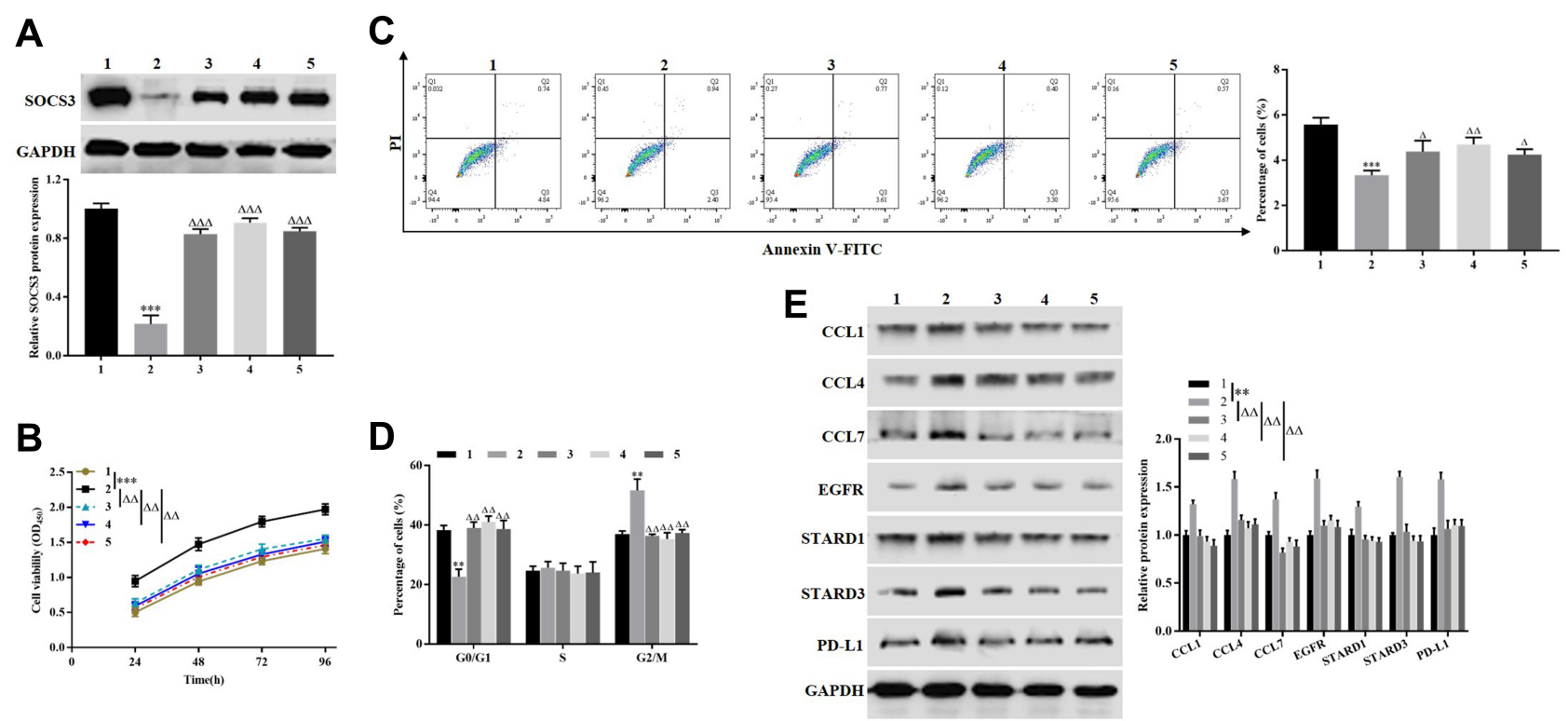

Figure 7 Combination therapy of FU and OXA inhibited the malignant biological behavior of HCTI I6 cells by downregulating PD-LI via miR-I83-5p/SOCS3 axis. (A and E) Western blotting was used to detect the expression of SOCS3, CCLI, CCL4, CCL7, EGFR, STARDI, STARD3 and PD-LI. (B) CCK-8 assay was employed to measure the proliferation of HCTII6 cells. (C and D) Flow cytometry was performed to assess the apoptosis and cell cycle of $\mathrm{HCTII6}$ cells. $* * \mathrm{P}<0.0 \mathrm{I}$, $* * * \mathrm{P}<0.00 \mathrm{I}$, vs. control group; ${ }^{\Delta} \mathrm{P}<0.05,{ }^{\Delta \Delta} \mathrm{P}<0.0 \mathrm{I},{ }^{\Delta \Lambda \Delta} \mathrm{P}<0.00$ I, vs. si-SOCS3 group. I: control group; 2: si-SOCS3 group; 3: si-SOCS3 + miR-I83-5p inhibitor group; 4: si-SOCS3 + si-PD-LI group; 5: si$\mathrm{SOCS} 3+\mathrm{FU}+\mathrm{OXA}$ group. 
demonstrated that combination of OXA and FU repressed the malignant biological behavior of HCT116 cells by downregulating PD-L1 through miR-183-5p/SOCS3 axis.

\section{Discussion}

As previously mentioned, PD-L1 was upregulated in a variety of malignancies and mediate the immune escape of tumor cells by inactivating $\mathrm{T}$ cells, which bring about a poor clinical outcome. ${ }^{17}$ Therefore, targeted PD-L1 therapies bring hope for the therapy of cancer. Considering that $\mathrm{CRC}$ is a heterogeneous tumor, a combination of FU and OXA was carried out to investigate the regulation mechanism of FU and OXA on PD-L1 expression, as well as on the development of CRC through PD-L1. Our results demonstrated that the combination of FU and OXA dramatically reduced the expression of PDL1. Also, the combination of FU and OXA notably inhibited cell proliferation, promoted cell apoptosis and arrest cells in G0/G1 phrase of HCT116 cells, as well as downregulated the expression of chemokines (CCL1, CCL4 and CCL7) and immune escape related proteins (EGFR, STARD1 and STARD3). These data illustrated that FU and OXA inhibited the malignant biological behavior of HCT116 cells via downregulating PD-L1.

MicroRNAs (miRNAs) are non-coding RNAs, highly conserved, and 18 $25 \mathrm{nt}$, participated in the regulation of cell growth, apoptosis and angiogenesis, and so on. ${ }^{18-20}$ Abnormal expression of miRNAs is taking part in various malignant biological behaviors of tumors. In addition, it had been reported that chemotherapeutic drugs can influence tumor progression through regulating miRNAs. ${ }^{10,21}$ Such as, Yang et al expounded that miR-214 was decreased in 5-FU-resistant CRC cells and overexpression of miR-214 increased the sensitivity of CRC cells to 5 -FU. ${ }^{22}$ OXA induced downregulation of miR-34a in CRC cells and overexpression of miR-34a weakened OXA resistance of CRC cells through TGF- $\beta / \mathrm{Smad} 4$ signaling pathway. ${ }^{23}$ In our article, we expounded that miR183-5p was upregulated in HCT116 cells and decreased after treated with FU and OXA. Functional experimental confirmation that silencing miR-183-5p also repressed cell viability and expression of chemokines, immune escape related proteins, as well as PD-L1, facilitated cell apoptosis and arrest cells in G0/G1 phrase.

SOCS3 is a member of the SOCS family which participates in the process of cell biology through a negative feedback loop. ${ }^{24}$ Studies have confirmed that SOCS3, as a signal suppressor, inhibits tumor cells proliferation, causes G1 arrest by suppressing JAK2/STAT3 signaling pathway. ${ }^{25}$ In CRC tissues, SOCS3 expression was decreased, and SOCS3 overexpression repressed biological behavior of CRC cells by negative regulating IL6/ STAT3 signaling pathway. ${ }^{26}$ Furthermore, Hirahara et al demonstrated that silencing SOCS3 could result in increase of PD-L1 expression in a STAT1-dependent manner. ${ }^{27}$ Zhang et al described that overexpression of SOCS3 could facilitate Th17 differentiation via suppressing PD-L1 upregulation. ${ }^{28}$ In this research, we found that SOCS3 overexpression suppressed the expression of PDL1, whereas knockdown of SOCS3 had opposite result. At the same time, miR-183-5p overexpression remitted PDL1 downregulation induced by SOCS3 overexpression via targeting downregulated SOCS3. Further experiments found that silencing SOCS3 promoted cell proliferation and the expression of chemokines and immune escape related proteins, inhibited cell apoptosis and facilitated cells in G2/M phrase. Treating HCT116 cells which silencing SOCS3 with FU and OXA could restore the promotion effect of knockdown of SOCS3 on the malignant biological behavior of HCT116 cells. Knockdown of SOCS3 and miR-183-5p or PD-L1 simultaneously showed the same results. All these results confirmed that the combination of FU and OXA suppressed the progression of CRC by inhibiting PD-L1 expression through miR-183-5p/ SOCS3 axis.

\section{Conclusion}

The combination therapy of OXA and FU could suppress the malignant biological behavior, and the mechanism was realized by inhibiting PD-L1 through miR-183-5p/SOCS3 axis.

\section{Acknowledgments}

Project funding for this study was received from Joint Fund of Yunnan Provincial Department of Science and Technology and Kunming Medical University 2019FE001(-073), 2018FE001(-069).

\section{Disclosure}

The authors report no conflicts of interest in this work.

\section{References}

1. Dienstmann R, Salazar R, Tabernero J. Personalizing colon cancer adjuvant therapy: selecting optimal treatments for individual patients. J Clin Oncol. 2015;33(16):1787-1796. doi:10.1200/JCO.2014.60.0213 
2. Treasure T, Monson K, Fiorentino F, et al. The CEA Second-Look Trial: a randomised controlled trial of carcinoembryonic antigen prompted reoperation for recurrent colorectal cancer. BMJ Open. 2014;4(5):e004385. doi:10.1136/bmjopen-2013-004385

3. Llosa NJ, Luber B, Siegel N, et al. Immunopathologic stratification of colorectal cancer for checkpoint blockade immunotherapy. Cancer Immunol Res. 2019;7(10):1574-1579. doi:10.1158/2326-6066.CIR18-0927

4. Franke AJ, Skelton WP, Starr JS, et al. Immunotherapy for colorectal cancer: a review of current and novel therapeutic approaches. $J$ Natl Cancer Inst. 2019;111(11):1131-1141. doi:10.1093/jnci/djz093

5. Oliveira AF, Bretes L, Furtado I. Review of PD-1/PD-L1 inhibitors in metastatic dMMR/MSI-H colorectal cancer. Front Oncol. 2019;9:396. doi:10.3389/fonc.2019.00396

6. Filippova N, Yang X, An Z, et al. Blocking PD1/PD-L1 interactions together with MLN4924 therapy is a potential strategy for glioma treatment. J Cancer Sci Ther. 2018;10(8):190-197. doi:10.4172/ 1948-5956.1000543

7. Kythreotou A, Siddique A, Mauri FA, et al. PD-L1. J Clin Pathol. 2018;71(3):189-194. doi:10.1136/jclinpath-2017-204853

8. Llosa NJ, Cruise M, Tam A, et al. The vigorous immune microenvironment of microsatellite instable colon cancer is balanced by multiple counter-inhibitory checkpoints. Cancer Discov. 2015;5(1):43-51. doi:10.1158/2159-8290.CD-14-0863

9. Gasiule S, Dreize N, Kaupinis A, et al. Molecular insights into miRNA-driven resistance to 5-fluorouracil and oxaliplatin chemotherapy: miR-23b modulates the epithelial-mesenchymal transition of colorectal cancer cells. J Clin Med. 2019;8(12):2115. doi: $10.3390 / \mathrm{jcm} 8122115$

10. Liu T, Zhang X, Du L, et al. Exosome-transmitted miR-128-3p increase chemosensitivity of oxaliplatin-resistant colorectal cancer. Mol Cancer. 2019;18(1):43. doi:10.1186/s12943-019-0981-7

11. Wang G, Zhou J, Lu F, et al. Downregulation of microRNA-183-5p inhibits the proliferation and invasion of colorectal cancer cells by inactivating the reticulocalbin-2/Wnt/ $\beta$-catenin signaling pathway. Mol Med Rep. 2019;19(5):4475-4483. doi:10.3892/mmr.2019.10059

12. Zheng S, Zhong YF, Tan DM, et al. miR-183-5p enhances the radioresistance of colorectal cancer by directly targeting ATG5. J Biosci. 2019;44(4). doi:10.1007/s12038-019-9918-y

13. Xu JZ, Shao CC, Wang XJ, et al. circTADA2As suppress breast cancer progression and metastasis via targeting miR-203a-3p/SOCS3 axis. Cell Death Dis. 2019;10(3):175. doi:10.1038/s41419-019-1382-y

14. Li M, Zheng R, Yuan FL. MiR-410 affects the proliferation and apoptosis of lung cancer A549 cells through regulation of SOCS3/ JAK-STAT signaling pathway. Eur Rev Med Pharmacol Sci. 2018;22 (18):5987-5993. doi:10.26355/eurrev_201809_15933
15. Hamilton KE, Lund PK, J A G, et al. Suppressor of cytokine signaling 3 (SOCS3) is not an independent biomarker of colorectal adenoma risk. BMC Res Notes. 2010;3:144. doi:10.1186/1756-05003-144

16. Wang J, Guo J, Fan H. MiR-155 regulates the proliferation and apoptosis of pancreatic cancer cells through targeting SOCS3. Eur Rev Med Pharmacol Sci. 2019;23(12):5168-5175. doi:10.26355/ eurrev_201906_18181

17. Anitei MG, Zeitoun G, Mlecnik B, et al. Prognostic and predictive values of the immunoscore in patients with rectal cancer. Clin Cancer Res. 2014;20(7):1891-1899. doi:10.1158/1078-0432.CCR-13-2830

18. Correia de Sousa M, Gjorgjieva M, Dolicka D, et al. Deciphering miRNAs' action through miRNA editing. Int J Mol Sci. 2019;20(24). doi:10.3390/ijms20246249

19. Lu TX, Rothenberg ME. MicroRNA. J Allergy Clin Immunol. 2018;141(4):1202-1207. doi:10.1016/j.jaci.2017.08.034

20. Lin S, Gregory RI. MicroRNA biogenesis pathways in cancer. Nat Rev Cancer. 2015;15(6):321-333. doi:10.1038/nrc3932

21. Li J, Lu M, Jin J, et al. miR-449a suppresses tamoxifen resistance in human breast cancer cells by targeting ADAM22. Cell Physiol Biochem. 2018;50(1):136-149. doi:10.1159/000493964

22. Yang Y, Bao Y, Yang GK, et al. MiR-214 sensitizes human colon cancer cells to 5-FU by targeting Hsp27. Cell Mol Biol Lett. 2019;24:22. doi:10.1186/s11658-019-0143-3

23. Sun C, Wang FJ, Zhang HG, et al. miR-34a mediates oxaliplatin resistance of colorectal cancer cells by inhibiting macroautophagy via transforming growth factor- $\beta / \mathrm{Smad} 4$ pathway. World $J$ Gastroenterol. 2017;23(10):1816-1827. doi:10.3748/wjg.v23.i10.1816

24. Yoshimura A, Naka T, Kubo M. SOCS proteins, cytokine signalling and immune regulation. Nat Rev Immunol. 2007;7(6):454-465. doi:10.1038/nri2093

25. Singh S, Chouhan S, Mohammad N, et al. Resistin causes G1 arrest in colon cancer cells through upregulation of SOCS3. FEBS Lett. 2017;591(10):1371-1382. doi:10.1002/1873-3468.12655

26. Chu Q, Shen D, He L, et al. Prognostic significance of SOCS3 and its biological function in colorectal cancer. Gene. 2017;627:114-122. doi:10.1016/j.gene.2017.06.013

27. Hirahara K, Ghoreschi K, Yang XP, et al. Interleukin-27 priming of $\mathrm{T}$ cells controls IL-17 production in trans via induction of the ligand PD-L1. Immunity. 2012;36(6):1017-1030. doi:10.1016/j. immuni.2012.03.024

28. Zhang Y, Ma CA, Lawrence MG, et al. PD-L1 up-regulation restrains Th17 cell differentiation in STAT3 loss- and STAT1 gain-of-function patients. J Exp Med. 2017;214(9):2523-2533. doi:10.1084/ jem.20161427

\section{Publish your work in this journal}

Cancer Management and Research is an international, peer-reviewed open access journal focusing on cancer research and the optimal use of preventative and integrated treatment interventions to achieve improved outcomes, enhanced survival and quality of life for the cancer patient.
The manuscript management system is completely online and includes a very quick and fair peer-review system, which is all easy to use. Visit http://www.dovepress.com/testimonials.php to read real quotes from published authors. 